\title{
PROPRIEDADES DE CHAPAS DE FLOCOS FABRICADAS COM ADESIVO DE URÉIA-FORMALDEÍDO E DE TANINOS DA CASCA DE Eucalyptus grandis W. Hill ex Maiden OU DE Eucalyptus pellita F. Muell. ${ }^{1}$
}

\author{
Angélica de Cássia Oliveira Carneiro², Benedito Rocha Vital ${ }^{3}$, Alexandre Santos Pimenta ${ }^{3}$, Ricardo Marius \\ Della Lucia ${ }^{3}$
}

\begin{abstract}
RESUMO - Os taninos foram extraídos da casca de Eucalyptus grandis e Eucalyptus pellita, com água quente, à qual se adicionaram $4,5 \%$ de sulfito de sódio, durante três horas. As temperaturas da solução foram iguais a 70 e $100^{\circ} \mathrm{C}$ para Eucalyptus grandis e Eucalyptus pellita, respectivamente. Para a produção dos adesivos e com o intuito de reduzir a sua viscosidade, os taninos foram sulfitados com sulfito de sódio e ácido acético. Formulações adesivas foram preparadas adicionando-se 0, 25, 50, 75 ou 100\% de adesivos tânicos ao adesivo comercial de uréia-formaldeído. Foram fabricadas chapas de flocos de Pinus elliottii Engelm. e Eucalyptus grandis, utilizando-se $8 \%$ da formulação adesiva. As propriedades das chapas foram determinadas segundo a norma ASTM D-1037, de 1993. Observou-se que as propriedades das chapas foram superiores ao mínimo estabelecido pela norma ANSI/A 280.1-93, exceto no caso da resistência à umidade. Verificou-se, ainda, que o emprego de uma formulação adesiva contendo resina à base de uréia-formaldeído e tanino-formaldeído pode melhorar algumas propriedades.
\end{abstract}

Palavras-chave: taninos condensados, cascas, Eucalipto, adesivos para madeira, sulfitação.

\section{PROPERTIES OF FLAKEBOARDS MADE FROM UREA-FORMALDEHYDE AND BARK TANNINS ADHESIVES OF Eucalyptus grandis OR Eucalyptus pellita}

\begin{abstract}
Bark tannins of Eucalyptus grandis and Eucalyptus pellita were extracted with 4,5\% sodium sulfite in hot water solution for a period of three hours. Solution temperatures were 70 and $100^{\circ} C$, for Eucalyptus grandis and Eucalyptus pellita bark respectively. Tannins were reacted with acetic acid and sodium sulfite to reduce adhesive viscosity. Adhesive formulations were prepared adding $0,25,50,75$ or $100 \%$ of tannin adhesives to the commercial urea-formaldehyde adhesive. Flakeboards were fabricated with $8 \%$ resin content. Board properties were determined according to ASTM D-1037 standards. Except for humidity, all board properties were superior to the values established by ANSI.A 208.1-93 commercial standard. Addition of tannins to the urea-formaldehyde adhesive improved some properties.
\end{abstract}

Key-words: condensed tannins, barks, Eucalyptus, wood adhesives, sulfitation.

\footnotetext{
${ }^{1}$ Recebido para publicação em 16.6.2003 e aceito para publicação em 10.8.2004.

${ }^{2}$ Programa de Pós-Graduação em Ciência Florestal da UFV. E-mail: <o.carneiro@bol.com.br>.

${ }^{3}$ Departamento de Engenharia Florestal da UFV. E-mail: <bvital@ufv.br>.
} 


\section{INTRODUÇÃO}

Adesivo é um componente importante, com implicações técnicas e econômicas significativas na utilização da madeira (PIZZI, 1983), e seu custo pode chegar a 50\% do preço total desta. A qualidade da adesão, que afeta as propriedades das chapas, é determinada por vários fatores, mas principalmente pelo tipo e quantidade do adesivo (MOSLEMI, 1974; MALONEY, 1993). Assim, o desenvolvimento da indústria de painéis à base de madeira sempre acompanhou a evolução dos adesivos (DUNKY, 2001). No entanto, as pesquisas e desenvolvimentos relativos aos adesivos sempre foram motivados pela exigência de adesão adequada, pelo processo de produção e pelas propriedades dos painéis produzidos (DUNKY, 2001).

Atualmente, entre as principais necessidades da indústria de painéis à base de madeira, no que se refere a produção e desenvolvimento dos adesivos, citam-se redução do tempo de prensagem, melhor comportamento higroscópico dos painéis e adesivos alternativos e mais baratos (DUNKY, 2001).

Uma matéria-prima alternativa para a produção de adesivos com características fenólicas são os taninos (PIZZI, 1983). Estes são polifenóis de elevado peso molecular, encontrados nas cascas de plantas arbóreas, estando também freqüentemente presentes na madeira (PIZZI e MITTAL, 1994). Além de reagirem facilmente com o formaldeído, os taninos são substâncias renováveis, cuja extração pode incorporar valores à atividade florestal e minimizar o problema de descarte das cascas pela indústria madeireira.

Em alguns países como a África do Sul, a produção de adesivos tânicos empregando os taninos extraídos da casca de Acacia mearnsii De Wild. ocorre em escala industrial. No Brasil, a casca dessa espécie é, também, utilizada em escala industrial para a produção de taninos, enquanto a madeira é utilizada como lenha, carvão e matéria-prima para a produção de celulose, papel e chapas de fibra (SANTOS, 1997).

Os taninos encontrados na casca de outras espécies podem, também, ser utilizados para a fabricação de adesivos para madeira. No Brasil, um gênero promissor é o Eucalyptus Mori (2000), amplamente cultivado no país, principalmente no Estado de Minas Gerais. A madeira é normalmente empregada para a produção de celulose, carvão, chapas de composição e, mais recentemente, para a produção de madeira serrada. Em todos esses processos industriais, a casca forma um resíduo que normalmente é queimado para a geração de energia ou é descartado, poluindo o meio ambiente. Contudo, o emprego de taninos no preparo de adesivos para madeira tem sido limitado dada a sua elevada viscosidade, que reduz a sua vida útil e evita a formação de ligações cruzadas intramoleculares, diminuindo a resistência da linha de cola (SANTANA et al., 1995).

Uma alternativa para melhorar a qualidade dos adesivos tânicos é modificar os taninos através de hidrólise alcalina ou ácida (PIZZI, 1978). Além disso, junto com os taninos são extraídos gomas e açúcares que, também, prejudicam a qualidade dos adesivos (PIZZI e MITTAL, 1994). A hidrólise ácida ou alcalina reduz o efeito dessas substâncias, permitindo o preparo de formulações adesivas menos viscosas. $\mathrm{O}$ emprego dos taninos da casca de eucalipto para fabricação de adesivos é promissor, principalmente quando se trabalha com taninos hidrolisados e sulfitados (FETCHAL e RIEDL, 1993; MORI, 2000).

Assim o presente trabalho teve como objetivo geral avaliar as propriedades das chapas de flocos fabricadas com adesivo de uréia-formaldeído, ao qual foram adicionadas quantidades crescentes de adesivos tânicos produzidos com taninos hidrolisados e sulfitados e extraídos da casca de Eucalyptus grandis W. Hill ex Maiden e Eucalyptus pellita F. Muell.

\section{MATERIAL E MÉTODOS}

Os taninos foram extraídos de cascas de Eucalyptus grandis W. Hill ex Maiden, provenientes dos plantios da Universidade Federal de Viçosa, em Viçosa, MG, com idade de 20 anos e das cascas de Eucalyptus pellita $\mathrm{F}$. Muell. provenientes de Bom Despacho, MG (CAF), com idade de 17 anos.

A extração dos taninos foi feita empregando-se uma solução aquosa contendo 4,5\% de sulfito de sódio em relação à massa seca de casca. Essa porcentagem foi estabelecida em testes preliminares (CARNEIRO, 2002) e produz melhor rendimento gravimétrico em taninos. As extrações foram feitas em autoclave, utilizando-se temperatura de $70^{\circ} \mathrm{C}$ no caso de Eucalyptus grandis e $100^{\circ} \mathrm{C} \mathrm{em} \mathrm{Eucalyptus} \mathrm{pellita,} \mathrm{por} \mathrm{um} \mathrm{pe-}$ ríodo de três horas. Usou-se uma relação volume de licor/massa de casca de 20:1 em Eucalyptus grandis 
e de 30:1 em Eucalyptus pellita.

A sulfitação foi realizada em um balão de reação, onde foram colocados sob agitação mecânica constante e aquecimento: $50 \mathrm{~g}$ de taninos (massa seca), $50 \mathrm{~g}$ de água destilada, $2,5 \mathrm{~mL}$ de ácido acético e $5 \%$ de sulfito de sódio em relação à massa seca de taninos. Depois, essa mistura foi mantida sob refluxo durante 90 minutos. Para a sulfitação dos taninos de Eucalyptus pellita, utilizaram-se $75 \mathrm{~g}$ de água.

Os adesivos tânicos foram produzidos adicionandose aos taninos sulfitados $10 \%$ de formaldeído. Os adesivos tânicos assim preparados foram adicionados ao adesivo à base de uréia-formaldeído comercial (Cascamite PB 2346, ALBA-Química), nas proporções de $0,25,50,75$ e $100 \%$.

O arranjo experimental foi constituído de duas fontes de taninos, quatro teores de adição do adesivo tânico ao adesivo uréico e duas espécies de madeira, além de duas chapas fabricadas apenas com adesivo à base de uréia-formaldeído. Todas as chapas foram fabricadas em duplicata.

Os tratamentos ficaram constituídos conforme mostrado no Quadro 1.

Fabricaram-se chapas com $8 \%$ de adesivos à base de uréia-formaldeído (Cascamite PB 2346) e adesivos à base de taninos sulfitados, em relação à massa anidra de partículas. Utilizaram-se flocos com espessura de $0,5 \mathrm{~mm}$, largura de $2,5 \mathrm{~cm}$ e 7,0 cm de comprimento, produzidos no laboratório com madeira de Pinus elliottii (densidade: $0,40 \mathrm{~g} / \mathrm{cm}^{3}$ ) e Eucalyptus grandis (densidade: $0,56 \mathrm{~g} / \mathrm{cm}^{3}$ ). As chapas foram fabricadas com dimensões finais de 40,0 x 40,0 x 1,0 cm.

Empregou-se uma pressão nos pratos de $32 \mathrm{kgf} /$ $\mathrm{cm}^{2}$, temperatura de prensagem de $170{ }^{\circ} \mathrm{C}$, tempo de oito minutos e 30 segundos e uma razão de compactação de 1,4. Teve-se como meta uma densidade nominal igual a $0,56 \mathrm{~g} / \mathrm{cm}^{3} \mathrm{em}$ chapas de Pinus elliottii e 0,70 $\mathrm{g} / \mathrm{cm}^{3}$ nas chapas de eucalipto.

As propriedades físicas e mecânicas das chapas foram determinadas de acordo com a norma americana ASTM (1993). A resistência ao arrancamento de parafuso foi determinada na face. Os valores médios observados foram comparados com os mínimos exigidos pela norma comercial ANSI/A-208.1-1993 e pelas normas CSA 0437-93 e DIN 68761 (1) - 1961, na absorção e inchamento, uma vez que a norma ANSI/A não contempla essas especificações. Os valores exigidos por essas normas podem ser observados no Quadro 2 .

Quadro 1 - Tratamentos utilizados para fabricação das chapas Table 1 - Treatments as used for board fabrication

\begin{tabular}{|c|c|c|c|}
\hline Tratamento & Tipo de Adesivo Taninos/Uréia & Composição de Adesivo (\%) & Espécie de Madeira \\
\hline 1 & Eucalyptus grandis/uréia & $100 / 0$ & Pinus elliottii \\
\hline 2 & Eucalyptus pellita/uréia & $100 / 0$ & Pinus elliottii \\
\hline 3 & Eucalyptus grandis/uréia & $75 / 25$ & Pinus elliottii \\
\hline 4 & Eucalyptus pellita /uréia & $75 / 25$ & Pinus elliottii \\
\hline 5 & Eucalyptus grandis/uréia & $50 / 50$ & Pinus elliottii \\
\hline 6 & Eucalyptus pellita /uréia & $50 / 50$ & Pinus elliottii \\
\hline 7 & Eucalyptus grandis/uréia & $25 / 75$ & Pinus elliottii \\
\hline 8 & Eucalyptus pellita /uréia & $25 / 75$ & Pinus elliottii \\
\hline 9 & Uréia & 100 & Pinus elliottii \\
\hline 10 & Eucalyptus grandis/uréia & $100 / 0$ & Eucalyptus grandis \\
\hline 11 & Eucalyptus pellita/uréia & $100 / 0$ & Eucalyptus grandis \\
\hline 12 & Eucalyptus grandis/uréia & $75 / 25$ & Eucalyptus grandis \\
\hline 13 & Eucalyptus pellita /uréia & $75 / 25$ & Eucalyptus grandis \\
\hline 14 & Eucalyptus grandis/uréia & $50 / 50$ & Eucalyptus grandis \\
\hline 15 & Eucalyptus pellita /uréia & $50 / 50$ & Eucalyptus grandis \\
\hline 16 & Eucalyptus grandis/uréia & $25 / 75$ & Eucalyptus grandis \\
\hline 17 & Eucalyptus pellita /uréia & $25 / 75$ & Eucalyptus grandis \\
\hline 18 & Uréia & 100 & Eucalyptus grandis \\
\hline
\end{tabular}


Quadro 2 - Propriedades requeridas para chapas de composição

Table 2 - Composition boards grade requirements

\begin{tabular}{|c|c|c|c|c|c|c|c|c|c|c|c|}
\hline \multirow{3}{*}{ Classific. } & \multirow{2}{*}{\multicolumn{3}{|c|}{$\begin{array}{c}\text { Inchamento } \\
\text { em Espessura } \\
\text { Tolerância Média } \\
\text { do Painel (\%) }\end{array}$}} & \multirow{3}{*}{$\begin{array}{r}\mathrm{Ab} \\
\text { Expansão } \\
\text { Linear }(\%)\end{array}$} & \multicolumn{2}{|c|}{$\begin{array}{l}\text { bsorção em Água } \\
\text { Tolerância (\%) } \\
\end{array}$} & \multicolumn{2}{|c|}{$\begin{array}{l}\text { Flexão Estática } \\
\text { de Parafuso (N) }\end{array}$} & \multirow{3}{*}{$\begin{array}{c}\text { Tração } \perp \\
\text { (Mpa) }\end{array}$} & \multicolumn{2}{|c|}{ Arrancamento } \\
\hline & & & & & & & \multirow[t]{2}{*}{$\begin{array}{l}\mathrm{MOE} \\
(\mathrm{MPa})\end{array}$} & \multirow[t]{2}{*}{$\begin{array}{l}\mathrm{MOR} \\
(\mathrm{MPa})\end{array}$} & & \multirow[t]{2}{*}{ Face } & \multirow[t]{2}{*}{ Borda } \\
\hline & Amb. & $2 \mathrm{~h}$ & $24 \mathrm{~h}$ & & $2 \mathrm{~h}$ & $24 \mathrm{~h}$ & & & & & \\
\hline $\mathrm{H}-1$ & $\mathrm{NE}$ & $\mathrm{NE}$ & $\mathrm{NE}$ & $\mathrm{NE}$ & $\mathrm{NE}$ & $\mathrm{NE}$ & 2400 & 16,5 & 0,9 & 1800 & 1320,5 \\
\hline $\mathrm{H}-2$ & $\mathrm{NE}$ & $\mathrm{NE}$ & $\mathrm{NE}$ & $\mathrm{NE}$ & NE & $\mathrm{NE}$ & 2400 & 20,5 & 0,9 & 1900 & 1550 \\
\hline $\mathrm{H}-3$ & $\mathrm{NE}$ & $\mathrm{NE}$ & $\mathrm{NE}$ & $\mathrm{NE}$ & $\mathrm{NE}$ & $\mathrm{NE}$ & 2750 & 23,5 & 1,0 & 2000 & 1550 \\
\hline$M-1$ & $\mathrm{NE}$ & $\mathrm{NE}$ & $\mathrm{NE}$ & 0,35 & $\mathrm{NE}$ & $\mathrm{NE}$ & 1725 & 11,0 & 0,4 & $\mathrm{NE}$ & $\mathrm{NE}$ \\
\hline$M-S$ & $\mathrm{NE}$ & $\mathrm{NE}$ & $\mathrm{NE}$ & 0,35 & $\mathrm{NE}$ & $\mathrm{NE}$ & 1900 & 12,5 & 0,4 & 800 & 800 \\
\hline$M-2$ & $\mathrm{NE}$ & $6 *$ & $15^{*}$ & 0,35 & $10 * *$ & $15 * *$ & 2250 & 14,5 & 0,45 & 900 & 900 \\
\hline$M-3$ & $\mathrm{NE}$ & $\mathrm{NE}$ & $\mathrm{NE}$ & 0,35 & $\mathrm{NE}$ & $\mathrm{NE}$ & 2750 & 16,5 & 0,55 & 1000 & 1000 \\
\hline $\mathrm{LD}-1$ & $\mathrm{NE}$ & 6 & 15 & 0,35 & 10 & 15 & 550 & 5,0 & 0,10 & $\mathrm{NE}$ & $\mathrm{NE}$ \\
\hline$L D-2$ & $\mathrm{NE}$ & $\mathrm{NE}$ & $\mathrm{NE}$ & 0,35 & $\mathrm{NE}$ & $\mathrm{NE}$ & 1025 & 5,0 & 0,15 & 550 & $\mathrm{NE}$ \\
\hline
\end{tabular}

$\mathrm{H}=$ alta densidade (superior a $\left.800 \mathrm{~kg} / \mathrm{m}^{3}\right), \mathrm{M}=$ média densidade $\left(640-800 \mathrm{~kg} / \mathrm{m}^{3}\right)$, MS $=$ densidade média especial (Propriedades

físicas entre M-1 e M-2), LD = baixa densidade (menor que $640 \mathrm{~kg} / \mathrm{m}^{3}$ ) e NE = não-especificado.

* Norma DIN 68761 (1) - 1961 e ** norma CSA 0437-1993.

Fonte: Adaptado de U.S. Forest Products Laboratory (1999).

Os resultados do experimento foram interpretados com o auxílio de análise de regressão, adotandose um nível de 5\% de significância. A escolha do melhor modelo foi baseada na significância estatística dos coeficientes de regressão, no maior coeficiente de determinação $\left(\mathrm{R}^{2}\right)$ e na análise dos resíduos.

\section{RESULTADOS E DISCUSSÕES}

Os valores médios das densidades estão listados no Quadro 3. Observa-se, nesse quadro, que a densidade média das chapas fabricadas com madeira de eucalipto foi inferior à desejada, provavelmente devido à aplicação de uma taxa de compressão menor que a necessária. Contudo, as chapas fabricadas com partículas da mesma espécie tiveram densidades homogêneas, uma vez que os valores médios de densidade não diferiram entre si, pelo teste $\mathrm{F}$ a $5 \%$ de probabilidade.

No Quadro 4 estão os valores médios da umidade de equilíbrio das chapas após seu condicionamento em meio ambiente com temperatura média de $25^{\circ} \mathrm{C}$ e umidade relativa de $65 \%$.

A análise de regressão indicou variações significativas na umidade de equilíbrio higroscópico, tan- to nas chapas fabricadas com flocos de Pinus elliottii quanto nas de Eucalyptus grandis, conforme os tratamentos. Os coeficientes das equações são apresentados no Quadro 5.

Conforme pode ser observado no Quadro 4 e analisando os coeficientes das equações apresentadas no Quadro 5, o aumento da porcentagem de taninos na formulação adesiva, de modo geral, ocasionou aumento na umidade de equilíbrio higroscópico de todas as chapas fabricadas. Nas chapas fabricadas com partículas de Pinus elliottii, não se verificaram diferenças decorrentes da espécie fornecedora dos taninos. Contudo, nas chapas fabricadas com partículas de eucalipto, os taninos de Eucalyptus grandis proporcionaram umidades de equilíbrio significativamente menores do que aqueles observados nos taninos de Eucalyptus pellita. Essas diferenças se devem à maior higroscopicidade dos taninos ou à presença de componentes hidrofílicos extraídos das cascas.

Os valores médios da resistência das chapas estão apresentados no Quadro 6, no caso das chapas fabricadas com partículas de Pinus elliottii e Eucalyptus grandis. No Quadro 7, mostram-se os coeficientes das equações obtidas por análise de regressão, bem como o coeficiente de determinação dessa regressão. 
Quadro 3 - Valores médios de densidade $\left(\mathrm{g} / \mathrm{cm}^{3}\right)$ em chapas de Pinus elliottii e Eucalyptus grandis Table 3 - Pinus elliottii and Eucalyptus grandis boards mean density $\left(\mathrm{g} / \mathrm{cm}^{3}\right)$

\begin{tabular}{ccccc}
\hline Tratamento & Tipo de Adesivo Tanino/Uréia & CAD $(\%)$ & Espécie de Madeira & ${\text { Densidade }\left(\mathrm{g} / \mathrm{cm}^{3}\right)^{*}}$ \\
\hline 1 & Eucalyptus grandis/uréia & $100 / 0$ & Pinus elliottii & 0,57 \\
2 & Eucalyptus pellita/uréia & $100 / 0$ & Pinus elliottii & 0,57 \\
3 & Eucalyptus grandis/uréia & $75 / 25$ & Pinus elliottii & 0,57 \\
4 & Eucalyptus pellita /uréia & $75 / 25$ & Pinus elliottii & 0,58 \\
5 & Eucalyptus grandis/uréia & $50 / 50$ & Pinus elliottii & 0,57 \\
6 & Eucalyptus pellita/uréia & $50 / 50$ & Pinus elliottii & 0,60 \\
7 & Eucalyptus grandis/uréia & $25 / 75$ & Pinus elliottii & 0,56 \\
8 & Eucalyptus pellita/uréia & $25 / 75$ & Pinus elliottii & 0,58 \\
9 & Uréia & 100 & Pinus elliottii & 0,56 \\
10 & Eucalyptus grandis/uréia & $100 / 0$ & Eucalyptus grandis & 0,67 \\
11 & Eucalyptus pellita/uréia & $100 / 0$ & Eucalyptus grandis & 0,69 \\
12 & Eucalyptus grandis/uréia & $75 / 25$ & Eucalyptus grandis & 0,70 \\
13 & Eucalyptus pellita/uréia & $75 / 25$ & Eucalyptus grandis & 0,67 \\
14 & Eucalyptus grandis/uréia & $50 / 50$ & Eucalyptus grandis & 0,67 \\
15 & Eucalyptus pellita/uréia & $50 / 50$ & Eucalyptus grandis & 0,69 \\
16 & Eucalyptus grandis/uréia & $25 / 75$ & Eucalyptus grandis & 0,68 \\
17 & Eucalyptus pellita/uréia & $25 / 75$ & Eucalyptus grandis & 0,67 \\
18 & Uréia & 100 & Eucalyptus grandis & 0,68 \\
\hline
\end{tabular}

Em que CAD = composição do adesivo e proporção de taninos em relação à uréia.

Não se observou diferença estatística, pelo teste $\mathrm{F}$ a $5 \%$ de probabilidade, entre as médias de densidades das chapas produzidas com a mesma espécie de madeira.

Quadro 4 - Valores médios da umidade de equilíbrio em chapas produzidas com madeira de Pinus elliottii e Eucalyptus grandis Table 4 - Equilibrium moisture content for Pinus elliottii and Eucalyptus grandis boards

\begin{tabular}{|c|c|c|c|c|c|c|c|}
\hline \multicolumn{4}{|c|}{ Chapas de Pinus elliottii } & \multicolumn{4}{|c|}{ Chapas de Eucalyptus grandis } \\
\hline Tratamento & CAD (\%) & Fonte Taninos & Umidade $(\%)$ & Tratamento & $\mathrm{CAD}(\%)$ & FonteTaninos & Umidade $(\%)$ \\
\hline 1 & $100 \mathrm{~T}$ & E. grandis & 10,1 & 10 & $100 \mathrm{~T}$ & E.grandis & 11,5 \\
\hline 2 & $100 \mathrm{~T}$ & E. pellita & 11,3 & 11 & $100 \mathrm{~T}$ & E.pellita & 12,0 \\
\hline 3 & $25 \mathrm{U} / 75 \mathrm{~T}$ & E. grandis & 11,1 & 12 & $25 \mathrm{U} / 75 \mathrm{~T}$ & E.grandis & 10,8 \\
\hline 4 & $25 \mathrm{U} / 75 \mathrm{~T}$ & E. pellita & 10,8 & 13 & $25 \mathrm{U} / 75 \mathrm{~T}$ & E. pellita & 10,9 \\
\hline 5 & $50 \mathrm{U} / 50 \mathrm{~T}$ & E.grandis & 10,3 & 14 & $50 \mathrm{U} / 50 \mathrm{~T}$ & E.grandis & 9,8 \\
\hline 6 & $50 \mathrm{U} / 50 \mathrm{~T}$ & E. pellita & 10,5 & 15 & $50 \mathrm{U} / 50 \mathrm{~T}$ & E.pellita & 10,4 \\
\hline 7 & $75 \mathrm{U} / 25 \mathrm{~T}$ & E. grandis & 10,4 & 16 & $75 \mathrm{U} / 25 \mathrm{~T}$ & E.grandis & 9,8 \\
\hline 8 & $75 \mathrm{U} / 25 \mathrm{~T}$ & E. pellita & 9,5 & 17 & $75 \mathrm{U} / 25 \mathrm{~T}$ & E.pellita & 10,1 \\
\hline 9 & $100 \mathrm{U}$ & 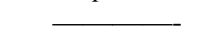 & 10,0 & 18 & $100 \mathrm{U}$ & ${ }^{2}$ & 9,3 \\
\hline
\end{tabular}

Em que $\mathrm{U}=$ adesivo de uréia, $\mathrm{T}=$ adesivo de taninos e CAD = composição de adesivo.

Quadro 5 - Coeficientes das equações da regressão da umidade de equilíbrio das chapas produzidas com madeira de Pinus elliottii e Eucalyptus grandis

Table 5 -Regression equations coefficients for equilibrium moisture content for boards fabricated with Pinus elliottii and Eucalyptus grandis wood

\begin{tabular}{lccccc}
\hline Espécie & Intercepto & CAD & ESPTAN & CAD $^{2}$ & R2 $^{-}$ \\
\hline Pinus elliottii & 10,92 & $-1,0$ & - & -- & 0,37 \\
Eucalyptus grandis & 11,86 & $-3,63$ & $-0,29$ & 1,34 & 0,92 \\
\hline
\end{tabular}

Em que CAD = composição de adesivo $(0,25,50,75$ e 100\% de substituição do adesivo de uréia). ESPTAN = fonte de taninos, sendo em Eucalyptus grandis ESPTAN igual a $1 \mathrm{e}$ em Eucalyptus pellita ESPTAN igual a 0. 
Quadro 6 - Valores médios de resistência à tração perpendicular, arrancamento de parafuso e flexão estática (MOE e MOR), em chapas produzidas com madeira de Pinus elliottii e Eucalyptus grandis

Table 6 - Mean values for internal bond, screw withdrawal and static bending resistance for boards fabricated with Pinus elliottii and Eucalyptus grandis wood

\begin{tabular}{|c|c|c|c|c|c|c|c|c|c|c|c|}
\hline \multirow[b]{2}{*}{ Trat. } & \multirow[b]{2}{*}{ CAD $(\%)$} & \multirow[b]{2}{*}{$\begin{array}{l}\text { Fonte de } \\
\text { Taninos/ } \\
\text { Adesivo }\end{array}$} & \multicolumn{4}{|c|}{ Chapas de Pinus elliottii } & \multirow[b]{2}{*}{ Trat. } & \multicolumn{4}{|c|}{ Chapas de Eucalyptus grandis } \\
\hline & & & $\begin{array}{l}\text { Tração } \\
(\mathrm{MPa})\end{array}$ & $\begin{array}{c}\text { Arrancamento } \\
\text { Parafuso } \\
\text { (N) }\end{array}$ & $\begin{array}{l}\mathrm{MOE} \\
(\mathrm{MPa})\end{array}$ & $\begin{array}{l}\text { MOR } \\
(\mathrm{MPa})\end{array}$ & & $\begin{array}{l}\text { Tração } \\
(\mathrm{MPa})\end{array}$ & $\begin{array}{c}\text { Arrancamento } \\
\text { Parafuso } \\
\text { (N) }\end{array}$ & $\begin{array}{l}\mathrm{MOE} \\
(\mathrm{MPa})\end{array}$ & $\begin{array}{r}\mathrm{MOR} \\
(\mathrm{MPa})\end{array}$ \\
\hline 1 & $100 \mathrm{~T}$ & $\begin{array}{c}\text { Eucalyptus } \\
\text { grandis }\end{array}$ & 0,69 & 1131,9 & 3640,90 & 30,87 & 10 & 0,41 & 1296,05 & 3263,27 & 22,97 \\
\hline 2 & $100 \mathrm{~T}$ & $\begin{array}{c}\text { Eucalyptus } \\
\text { pellita }\end{array}$ & 0,32 & 1210,3 & 3015,78 & 22,41 & 11 & 0,27 & 1141,70 & 2533,04 & 15,93 \\
\hline 3 & $25 \mathrm{U} / 75 \mathrm{~T}$ & $\begin{array}{c}\text { Eucalyptus } \\
\text { grandis }\end{array}$ & 0,45 & 1558,2 & 2995,65 & 23,34 & 12 & 0,45 & 1372,00 & 3227,07 & 23,22 \\
\hline 4 & $25 \mathrm{U} / 75 \mathrm{~T}$ & $\begin{array}{c}\text { Eucalyptus } \\
\text { pellita }\end{array}$ & 0,34 & 1190,7 & 3194,35 & 24,87 & 13 & 0,37 & 1455,30 & 2895,64 & 23,87 \\
\hline 5 & $50 \mathrm{U} / 50 \mathrm{~T}$ & $\begin{array}{c}\text { Eucalyptus } \\
\text { grandis }\end{array}$ & 0,93 & 1372,0 & 3919,05 & 34,46 & 14 & 0,92 & 1501,85 & 3706,16 & 32,23 \\
\hline 6 & $50 \mathrm{U} / 50 \mathrm{~T}$ & $\begin{array}{c}\text { Eucalyptus } \\
\text { pellita }\end{array}$ & 0,63 & 1788,5 & 3862,92 & 27,83 & 15 & 0,44 & 1249,50 & 3240,40 & 27,89 \\
\hline 7 & $75 \mathrm{U} / 25 \mathrm{~T}$ & $\begin{array}{c}\text { Eucalyptus } \\
\text { grandis }\end{array}$ & 0,93 & 1465,1 & 3582,48 & 26,54 & 16 & 1,08 & 1587,60 & 3183,44 & 28,06 \\
\hline 8 & $75 \mathrm{U} / 25 \mathrm{~T}$ & $\begin{array}{c}\text { Eucalyptus } \\
\text { pellita }\end{array}$ & 0,84 & 1504,3 & 4236,17 & 31,03 & 17 & 0,69 & 2156,00 & 2817,14 & 24,81 \\
\hline 9 & $100 \mathrm{U}$ & "Uréia" & 0,91 & 1489,6 & 3211,89 & 28,95 & 18 & 1,05 & 2072,70 & 3178,84 & 29,33 \\
\hline Norma & - & - & $>0,15$ & $>550$ & $>1025$ & $>5$ & - & $>0,40$ & $>900$ & 2250 & $>14,50$ \\
\hline
\end{tabular}

Em que $\mathrm{U}=$ adesivo de uréia, $\mathrm{T}$ = adesivo tânico e CAD = composição do adesivo.

Quadro 7 - Coeficientes das equações e coeficientes de determinações referentes às propriedades mecânicas das chapas produzidas com madeira de Pinus elliottii e Eucalyptus grandis

Table 7 - Equations coefficients and coefficients of determination related to properties of boards fabricated with Pinus elliottii and Eucalyptus grandis wood

\begin{tabular}{|c|c|c|c|c|c|c|}
\hline Propriedade & Intercepto & CAD & ESPTAN & $\mathrm{CAD}^{2}$ & $\mathrm{CAD}^{3}$ & $\mathrm{R} 2$ \\
\hline \multicolumn{7}{|c|}{ Chapas de Pinus elliottii } \\
\hline Tração & 0,340 & 0,51 & 0,17 & - & - & 0,73 \\
\hline Arranque & 1170 & 1096,9 & - & -798 & - & 0,48 \\
\hline MOE & 3301,6 & $-2821,9$ & - & 12015,9 & $-9310,4$ & 0,65 \\
\hline MOR & 24,58 & 8,28 & 1,81 & $-4,56$ & - & 0,20 \\
\hline \multicolumn{7}{|c|}{ Chapas de Eucalyptus grandis } \\
\hline Tração & 0,203 & 0,59 & 0,21 & 0,16 & - & 0,88 \\
\hline Arranque & 1272,2 & - & - & 847,7 & - & 0,74 \\
\hline MOE & 2710,9 & 1176,3 & 378,7 & $-976,2$ & - & 0,54 \\
\hline MOR & 18,04 & 23,38 & 2,79 & $-14,32$ & $\longrightarrow$ & 0,74 \\
\hline
\end{tabular}

Em que CAD = composição de adesivo $(25,50,75$ e 100\% de substituição do adesivo de uréia) e ESPTAN = fonte de taninos, sendo em Eucalyptus grandis ESPTAN igual a 1 e em Eucalyptus pellita ESPTAN igual a 0. 
Analisando os coeficientes das equações apresentadas no Quadro 7, observou-se que a resistência à tração perpendicular, de modo geral, foi diretamente proporcional à porcentagem do adesivo comercial na formulação adesiva em ambas as fontes de taninos. Os taninos de Eucalyptus grandis produziram chapas significativamente mais resistentes do que os taninos de Eucalyptus pellita.

Conforme pode ser observado no Quadro 6, todas as chapas fabricadas com flocos de Pinus elliottii apresentaram resistência à tração superior ao mínimo exigido pela norma ANSI/A 208-1-93. As chapas fabricadas com flocos de eucalipto e adesivo puro de taninos de Eucalyptus pellita (T11) e o tratamento cuja formulação adesiva continha $25 \%$ de uréia-formaldeído e $75 \%$ de taninos de Eucalyptus pellita (T13) não atingiram o mínimo exigido pela norma.

Entre os tratamentos produzidos com a mistura dos dois adesivos, a melhor resistência à tração perpendicular foi obtida nos tratamentos cuja formulação adesiva continha $75 \%$ de adesivo de uréia e $25 \%$ de taninos de Eucalyptus grandis em sua formulação, obtendo-se uma resistência média de 1,08 MPa, como pode ser visto no Quadro 6.

Analisando, ainda, o Quadros 6, pode-se constatar que os valores médios de módulo de ruptura foram superiores ao mínimo exigido pela norma ANSI/A-2081/93. As equações obtidas por regressão indicam que a fonte de taninos e a composição do adesivo afetaram o módulo de ruptura. Nas chapas produzidas com madeira de eucalipto, os tratamentos que resultaram em maiores valores de MOR foram naquelas fabricadas com formulações adesivas contendo taninos de Eucalyptus grandis.

As chapas confeccionadas com formulações adesivas contendo 50\% de taninos de Eucalyptus grandis e 50\% de uréia em sua composição apresentaram os melhores valores médios de módulo de ruptura, sendo, inclusive, superiores aos das chapas produzidas apenas com o adesivo de uréia comercial.

Também pode ser observado no Quadro 6 que todos os tratamentos atenderam ao mínimo exigido pela norma ANSI/A -208.1/93, no módulo de elasticidade. Nas chapas fabricadas com Pinus elliottii, o maior módulo de elasticidade foi obtido quando se utilizou uma formulação adesiva contendo $75 \%$ de uréia-formaldeí- do e $25 \%$ de taninos de Eucalyptus pellita. Nas fabricadas com flocos de Eucalyptus grandis, o maior MOE foi obtido quando a formação continha $50 \%$ de uréia-formaldeído e 50\% de taninos extraídos da casca de Eucalyptus grandis. Os módulos de elasticidade observados nas chapas fabricadas com adesivos à base de uréia-formaldeído (T9 e T18) foram, de modo geral, inferiores aos verificados nas chapas feitas com adesivo puro à base de taninos de Eucalyptus grandis.

De acordo com a análise de regressão apresentada no Quadro 7, conclui-se que o efeito dos tratamentos experimentais no módulo de elasticidade foi significativamente afetado pelas espécies de madeira utilizadas na produção das chapas, uma vez que naquelas produzidas com madeira de eucalipto a composição do adesivo apresentou relação diretamente proporcional ao teor de adesivo de uréia-formaldeído na composição do adesivo, enquanto nas chapas confeccionadas com madeira de Pinus elliottii essa relação foi inversamente proporcional. A fonte de taninos utilizada na elaboração dos adesivos afetou, de forma significativa, apenas as chapas feitas com E. grandis.

A resistência média ao arrancamento de parafuso (Quadro 6) na face da chapa foi superior ao mínimo estipulado pela norma ANSI/A 208.1-93, que exige, em chapas de baixa densidade, um valor de resistência igual ou superior a $550 \mathrm{~N}$ e, nas chapas de média densidade, resistência superior a $900 \mathrm{~N}$.

Os efeitos da composição do adesivo e da fonte de taninos utilizados podem ser analisados pelas equações descritas no Quadro 7. Observando as médias (Quadro 6) e interpretando os coeficientes das equações (Quadro 7), verificou-se que, de modo geral, à medida que aumentou a quantidade de adesivo uréiaformaldeído na composição do adesivo, aumentou também a resistência ao arranque, com algumas exceções, como no tratamento com $50 \%$ de taninos e $50 \%$ de uréia (T5), quando se utilizou madeira de Pinus elliottii. Constatou-se, pelas equações de regressão, que a fonte de taninos empregada não teve efeito significativo sobre a propriedade arrancamento de parafuso. Dentre os adesivos formulados com tanino-uréia/formaldeído, os melhores resultados foram obtidos pelos tratamentos possuindo adesivos de taninos de Eucalyptus pellita, em que os valores de resistência foram iguais a 1.788,5 $\mathrm{N}$ e $2156,0 \mathrm{~N}$.

R. Árvore, Viçosa-MG, v.28, n.5, p.715-724, 2004 
Os valores médios para inchamento em espessura, absorção de água e expansão linear em chapas produzidas com madeira de Pinus elliottii e Eucalyptus grandis estão apresentados no Quadro 8, enquanto os coeficientes das regressões, no Quadro 9.

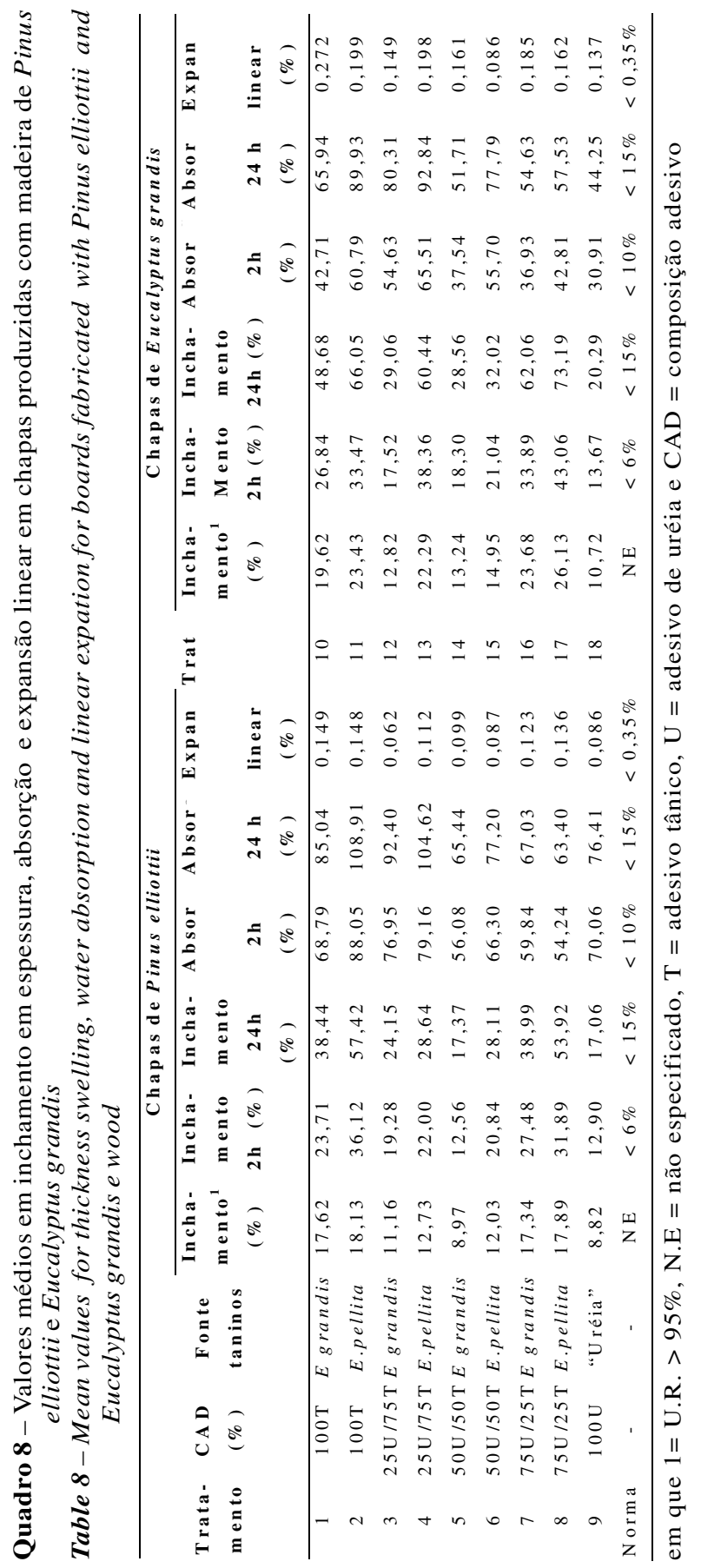

Todas as chapas apresentaram inchamento em espessura e absorção de água superiores àqueles permitidos pelas normas CSA 0437-93 e DIN (Quadro 1). Os elevados valores observados nesse experimento se devem, pelo menos em parte, ao fato de não ter sido utilizado parafina em emulsão no preparo das chapas.

De acordo com os resultados e pelas equações de análise de regressão, o inchamento em espessura e absorção foram, de modo geral, afetados pelo tipo de tanino utilizado nas formulações. Analisando exclusivamente os tratamentos quanto à fonte de taninos, pôde-se verificar que, mesmo ultrapassando os valores máximos permitidos para inchamento em espessura e absorção, após 2 e 24 horas de imersão em água as formulações que continham adesivos de taninos de Eucalyptus grandis incharam menos do que aquelas produzidas com taninos de Eucalyptus pellita. É provável que a maior absorção de água pelos tratamentos com Eucalyptus pellita seja devida à modificação química dos taninos, que os deixou mais higroscópicos. Quando se utilizou madeira de Pinus elliottii, as chapas fabricadas com formulação adesiva contendo 50\% $150 \%$ e $25 \% / 75 \%$ de taninos em relação à uréia-formaldeído foram as que menos incharam e absorveram, água respectivamente.

Salienta-se que, mesmo obtendo valores médios acima do mínimo permitido pela norma, as chapas fabricadas apenas com adesivos de uréia foram significativamente mais estáveis.

Todos os tratamentos atenderam às especificações exigidas pela norma ANSI/A.1.208.93, que permite uma expansão linear máxima de $0,35 \%$. Observase ainda, no Quadro 8, que as chapas produzidas com adesivos puros de taninos modificados e aquelas confeccionadas com $75 \%$ de adesivo de uréia $+25 \%$ de adesivo de taninos foram os tratamentos que obtiveram as maiores variações em expansão.

\section{RESUMO E CONCLUSÕES}

O presente trabalho teve como objetivo avaliar o efeito da adição de taninos sulfitados e extraídos da casca de Eucalyptus grandis e Eucalyptus pellita em formulações adesivas destinadas à fabricação de chapas de flocos. Os taninos foram extraídos com água quente, com adição de 4,5\% de sulfito de sódio, durante três horas, nas temperaturas de 70 e 100 ${ }^{\circ} \mathrm{C}$, respectivamente, em Eucalyptus grandis e Eucalyptus pellita. Para a produção dos adesivos, os taninos foram 
reagidos com ácido acético e sulfito de sódio por um período de 90 minutos. Essa reação fez-se necessária para a redução da viscosidade do adesivo.

Foram produzidas, em laboratório, chapas de flocos de Eucalyptus grandis W. Hill ex Maiden e Pinus elliottii, utilizando-se $8 \%$ de adesivo puro de taninos sulfitado extraído das cascas de Eucalyptus grandis e Eucalyptus pellita. Foram fabricadas, também, chapas com formulações adesivas tânicas, às quais foram adicionados $0,25,50,75$ e $100 \%$ de adesivo comercial à base de uréia-formaldeído, em relação à massa de adesivo tânico.

As propriedades das chapas foram determinadas segundo a norma ASTM D-1037 de 1993. Observouse que as propriedades das chapas produzidas com adesivo puro à base de taninos estavam acima do mínimo exigido pela norma comercial ANSI/A 208.1-93, com exceção das propriedades de resistência à umidade.

A combinação dos adesivos tânicos de eucalipto com os adesivos à base de uréia-formaldeído ocasionou aumento em algumas propriedades mecânicas.

Os resultados indicaram a potencialidade do uso dos taninos de eucalipto para a produção de adesivos a serem usados em chapas de composição para emprego em interiores, onde a resistência à umidade não é exigida.

Quadro 9 - Coeficientes das equações e coeficientes de determinação da regressão, do inchamento, em espessura e absorção de água, após 2 e 24 horas de imersão das chapas fabricadas com madeira de Pinus elliottii e Eucalyptus grandis Table 9 - Equations coefficients and coefficients of determination related to thickness swelling and water absorption after immersion in water for 2 and 24 hours for boards fabricated with Pinus elliottii and Eucalyptus grandis wood

\begin{tabular}{lcccccc}
\hline Propriedade & Intercepto & CAD & ESPTAN & CAD $^{2}$ & CAD $^{3}$ \\
\hline \multicolumn{7}{c}{ Chapas de Pinus elliottii } \\
\hline I. espessura (1) & 18,8 & $-66,6$ & $-1,13$ & 166,4 & $-108,8$ \\
I. espessura 2h (1) & 33,5 & $-112,7$ & $-5,50$ & 282,9 & $-187,1$ & 0,79 \\
I.espessura24h (1) & 54,1 & $-233,4$ & $-9,82$ & 581,2 & $-378,6$ & 0,74 \\
Absorção 2h (1) & 84,8 & $-48,0$ & $-5,22$ & - & 34,2 & 0,81 \\
Absorção 24h (1) & 107,1 & $-65,1$ & $-8,84$ & - & 0,58 \\
\hline \multicolumn{7}{c}{ Chapas de Eucalyptus grandis } \\
I. espessura (2) & 24,0 & $-71,8$ & $-3,48$ & 197,0 & $-136,0$ \\
I. espessura 2h (2) & 35,5 & $-95,9$ & $-7,87$ & 279,7 & $-200,2$ & 0,67 \\
I.espessura24h (2) & 66,4 & $-237,7$ & $-12,6$ & 642,4 & $-441,7$ & 0,56 \\
Absorção 2h (2) & 57,5 & 63,1 & $-10,6$ & $-188,3$ & 104,3 & 0,62 \\
Absorção 24h (2) & 85,3 & 70,8 & $-13,1$ & $-250,2$ & 145,6 & 0,86 \\
\hline
\end{tabular}

em que 1 = madeira Pinus elliottii, 2 = madeira de eucalipto, CAD = composição adesivo $(25,50,75$ e $100 \%$ de substituição do adesivo de uréia) e ESPTAN = fonte de taninos, sendo em Eucalyptus grandis ESPTAN igual a 1 e em Eucalyptus pellita ESPTAN, igual a 0 .

\section{REFERÊNCIAS BIBLIOGRÁFICAS}

AMERICAN NATIONAL STANDARD. ANS. Mat-formed wood particleboard: specification ANSI/A 208.1.1993. Gaithersburg: National Particleboards Association, 1993. 9p.

\section{AMERICAN SOCIETY FOR TESTING AND} MATERIALS - ASTM - Annual Book of ASTM Standards. Philadelphia: 1993.

CARNEIRO, A. C. O. Efeito da sulfitação dos taninos de $E$. grandis e $E$. pellita para produção de chapas de flocos. 2002. 90 f. Dissertação (Mestrado em Ciência Florestal), Universidade Federal de Viçosa. Viçosa, 2002.
CANADIAN STANDARTS ASSOCIATION. CSA. OSB and Waferboard. Ontario: CSA 0437 93, 1993. 18p.

DUNKY, M. Introduction. In: DUNKY, M. (Ed). Wood adhesion and glued products. COST Action E 13,Dynea: 2001. 161p.

\section{U.S. PRODUCTS LABORATORY. Wood}

handbook - Wood as an engineering material. Gen. Tech. Rep. FPL-GTR-113. Madison: U.S. Department of Agriculture, Forest Service,Forest Products Laboratory, 1999. 463 p.

GERMAN STANDARDS COMMITTEE (Deutschen Normanausschuss. 1971. Specifications for particleboard. DIN 68761 (1) 1961.Taschewbuch 1. Holz: 1961. 
MALONEY, T. M. Modern particleboard \& dry-process fiberboard manufacturing. San Francisco: Miller Freeman, 1993. 686 p.

MORI,F.A. Caracterização parcial dos taninos da casca e dos adesivos de três espécies de eucaliptos. 200. $73 \mathrm{f}$. Tese (Doutorado em Ciência Florestal) - Universidade Federal de Viçosa, Viçosa, 2000.

MOSLEMI, A. A. Particleboard. Carbondale: Southern Illinois University Press, 1974. v.1.

PIZZI, A. Wood adhesives: Chemistry and technology. New York: Marcell Dekker, 1983. 364p.
PIZZI, A.; MITTAL, K.L. Handbook of adhesive technology. New York: Marcell Dekker, 1994. 680p.

SANTANA, M.A.E.; BAUMANN, M.G.D.; CONNER, A.H. Resol resins prepared with tannin liquefied in phenol. Holzforschung, v.49, p 146-152, 1995

SANTOS, A. F. Caracterização da gomose da acácia-negra (Acácia mearnssi): I . Distribuição de lesões nos troncos. Curitiba: EMBRAPA-CNPF, 1997. 4P. 